\title{
Complete amelanotic acral nodular melanoma
}

\author{
Xingyu Mei MD, Zhouwei Wu MD PhD
}

Cite as: CMAJ 2018 October 15;190:E1231. doi: 10.1503/cmaj.180304

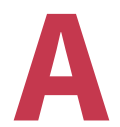

64-year-old woman presented to the dermatology department with a two-month history of a rapidly growing fleshy plaque on the sole of her left foot. Two months earlier, she had stood on a thorn that punctured the skin, and she subsequently noticed a "black spot" forming that was itchy.

On examination, the patient had a well-demarcated, nonpigmented plaque $\left(3 \times 1.5 \mathrm{~cm}^{2}\right.$ in diameter) with a granular spongy surface that was surrounded by a hyperkeratotic rim $(0.5 \mathrm{~cm}$ in width; Figure 1). Histopathological examination showed markedly atypical tumour cells proliferating in the dermis and epidermis (Figure 2). These tumour cells stained positively for S-100 protein and HMB-45 antigen, and negatively for cytokeratin and p63 antibody. DNA sequencing detected a BRAF V600E mutation in exon 15. Other laboratory examinations and positron emission tomography-computed tomography showed no evidence of spread or metastases.

We excised the lesion using a clinical margin of $2 \mathrm{~cm}$ and sent sentinel lymph nodes for biopsy. We diagnosed stage II B (T3bNOm0) melanoma. The guideline for melanoma from the National Comprehensive Cancer Network recommends either observation or treatment with interferon- $\alpha .{ }^{1}$ The patient declined treatment with interferon- $\alpha$ and was asked to follow up every three months. More than six months after surgery, she has no evidence of recurrence or metastasis.

Amelanotic melanoma is a subtype of melanoma with little or no black-to-brown pigmentation and represents $2 \%$ to $8 \%$ of all cases of melanoma. ${ }^{2}$ Complete amelanotic acral melanoma is uncommon and poses a diagnostic challenge to physicians because it often mimics other diseases. As in our case, the differential diagnosis includes pyogenic granuloma, eccrine poroma and its malignant counterpart porocarcinoma, or other infections that frequently appear on the sole of the foot.

Amelanotic acral melanoma occurs rarely; however, any nonhealing lesion requires careful evaluation that may include biopsy.

\section{References}

1. Coit DG, Thompson JA, Algazi A, et al. Melanoma, Version 2.2016, NCCN Clinical Practice Guidelines in Oncology. J Natl Compr Canc Netw 2016;14:450-73.

2. Jaimes N, Braun RP, Thomas L, et al. Clinical and dermoscopic characteristics of amelanotic melanomas that are not of the nodular subtype. J Eur Acad Dermatol Venereol 2012;26:591-6.

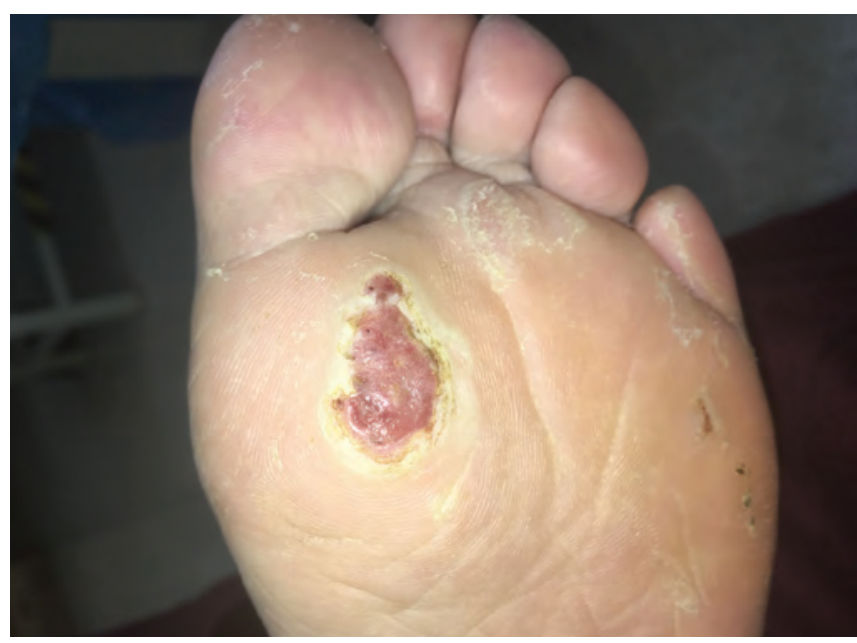

Figure 1: A nonpigmented plaque with a granular spongy surface and a hyperkeratotic rim found on the sole of the left foot of a 64-year-old woman.

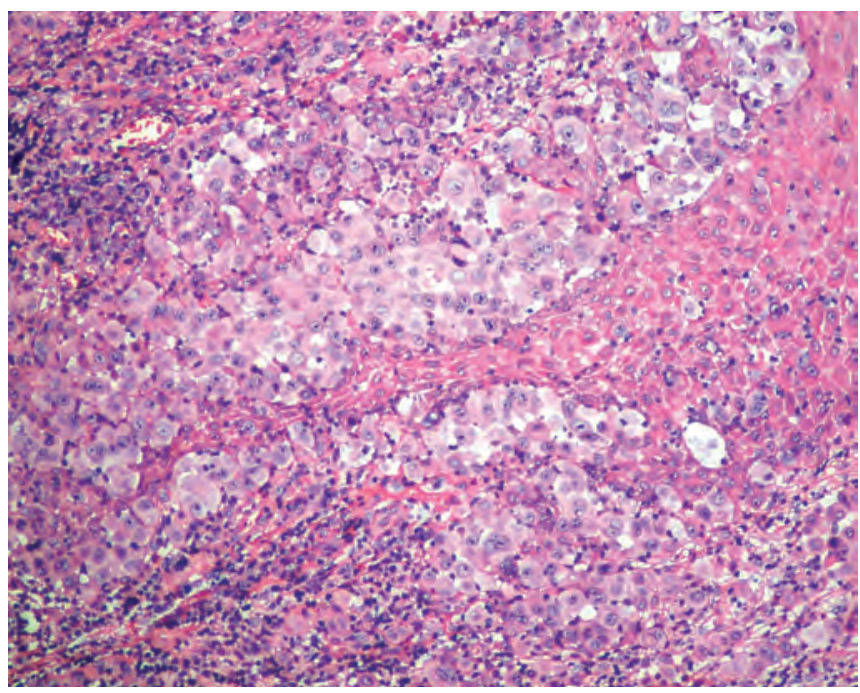

Figure 2: Histopathological examination of the lesion showing atypical melanocytic proliferation that is continuously invasive from the dermoepidermal junction through to the dermis. Hematoxylin and eosin stain. (Original magnification $\times 400$.)

\section{Competing interests: None declared.}

This article has been peer reviewed.

The authors have obtained patient consent.
Affiliation: Department of Dermatology, Shanghai First People's Hospital, Shanghai Jiaotong University, Shanghai, China

Correspondence to: Zhouwei Wu, 418950049@qq.com 